\title{
Effects of Noncommutativity on the Black Hole Entropy
}

\author{
Kumar S. Gupta, ${ }^{1}$ E. Harikumar, ${ }^{2}$ Tajron Jurić, ${ }^{3}$ Stjepan Meljanac, ${ }^{3}$ and Andjelo Samsarov ${ }^{3}$ \\ ${ }^{1}$ Theory Division, Saha Institute of Nuclear Physics, 1/AF Bidhannagar, Kolkata 700 064, India \\ ${ }^{2}$ School of Physics, University of Hyderabad, Central University P.O., Hyderabad 500046, India \\ ${ }^{3}$ Rudjer Bošković Institute, Bijenička c.54, 10002 Zagreb, Croatia \\ Correspondence should be addressed to Tajron Jurić; tjuric@irb.hr
}

Received 24 June 2014; Accepted 1 October 2014; Published 16 October 2014

Academic Editor: Anastasios Petkou

Copyright (C) 2014 Kumar S. Gupta et al. This is an open access article distributed under the Creative Commons Attribution License, which permits unrestricted use, distribution, and reproduction in any medium, provided the original work is properly cited. The publication of this article was funded by SCOAP S $^{3}$

The BTZ black hole geometry is probed with a noncommutative scalar field which obeys the $\kappa$-Minkowski algebra. The entropy of the BTZ black hole is calculated using the brick wall method. The contribution of the noncommutativity to the black hole entropy is explicitly evaluated up to the first order in the deformation parameter. We also argue that such a correction to the black hole entropy can be interpreted as arising from the renormalization of the Newton's constant due to the effects of the noncommutativity.

\section{Introduction}

Attempts to construct a quantum theory of gravity have a long history. At a macroscopic level, Einstein's theory of general relativity describes gravity in terms of spacetime geometry. It is therefore natural to expect that a quantum theory of gravity would lead to the modification of the classical spacetime structure and that the description of spacetime as a smooth manifold may no longer be valid at the Planck scale. Indeed, one of the approaches to quantum gravity takes this route and the spacetime is assumed to be noncommutative (NC) at the microscopic level [1]. Such an assumption is not ad hoc, since general relativity and Heisenberg's uncertainty principle together imply that the spacetime has a noncommutative structure $[2,3]$. With this motivation, different types of noncommutative space-times and their implications to physical models have been analyzed in recent times $[4,5]$.

Study of black hole physics plays an important role in exploring various quantum aspects of gravity. Even though black holes arose from the solutions of classical general relativity, many insights on the problem of semiclassical or quantum description of gravity were obtained from the study of field theories in black hole backgrounds. In particular, the aspect of black hole entropy and related thermodynamic properties [6-10] have been extensively studied in various frameworks including string theory [11, 12], loop gravity [13], conformal field theory [14-16], and some related approaches $[17,18]$. In the same spirit, there have been various attempts to construct noncommutative theories of gravity, noncommutative black hole solutions, and noncommutative quantum cosmology [19-30]. In particular, it has been shown that the noncommutative version of the BTZ black hole is described by a $\kappa$-deformed algebra $[31,32]$. Similar $\kappa$-deformed algebras have been found in the noncommutative description of Kerr black holes [33] and certain noncommutative versions of cosmology [22]. It thus appears that there is a certain element of universality in the appearance of the $\kappa$-deformed algebras, as they occur in the noncommutative (NC) descriptions of various types of classical geometries. It is therefore interesting to study the properties of black holes in the framework of $\kappa$ deformed noncommutative systems.

The $\kappa$-deformed Minkowski spacetime is defined by the algebra

$$
\left[\widehat{x}_{\mu}, \widehat{x}_{\nu}\right]=i\left(a_{\mu} \widehat{x}_{\nu}-a_{\nu} \widehat{x}_{\mu}\right) .
$$

Here, $a_{\mu}$ have dimensions of length and we choose $a_{0}=1 / \kappa \equiv$ $a$ and $a_{i}=0$ in the later part of this paper. Note that the rhs of $\kappa$-algebra is not a constant and is more general than that of 
the Moyal algebra. The symmetry algebra of this spacetime is known as $\kappa$-Poincaré-Hopf algebra [34]. Various aspects of this Hopf algebra have been studied in [35-39]. Each realization of the $\kappa$-algebra leads to a star product which can be used to construct the twisted coproduct, which ensures the invariance under twisted diffeomorphisms. Klein-Gordon theory in the $\kappa$-deformed spacetime was constructed [40, 41] and it was shown that the underlying Hopf algebra structure leads to twisted statistics $[42,43]$. The changes due to this twisted statistics of Klein-Gordon field near the vicinity of black hole were analyzed in [44]. The modification to Unruh effect due to $\kappa$-deformation of the spacetime was studied in $[45,46]$. Implication of the $\kappa$-deformation on electrodynamics was investigated in $[47,48]$, and deformed geodesic equation was obtained in [49]. In these papers, the approach adopted was to map the coordinates of the $\kappa$ deformed spacetime to that of commutative spacetime and using this map, functions of noncommutative coordinates were expressed in terms of commutative coordinates and their derivatives, as a perturbative expansion in powers of the deformation parameter. In these works, different realizations of mappings between noncommutative and commutative coordinates were used. This was done by embedding the $\kappa$ Minkowski spacetime algebra into Heisenberg algebra [50, 51]. In this paper, we study the change in the entropy of the BTZ black hole due to the $\kappa$-deformation of the spacetime. This is done by analyzing the $\kappa$-deformed Klein-Gordon field theory in the BTZ black hole background.

In $[6,7]$, a parallel between properties of black holes and thermodynamic variables in $3+1$ dimensions was obtained. By analyzing quantum field theory in the black hole background, Hawking showed that black holes emit thermal radiation and further the area law of black holes was derived [8]. Using these results and applying well known notions of quantum mechanics, 't Hooft had shown that there is a divergence in the allowed energy levels of a quantum mechanical particle near the black hole horizon. But the gravitational effects close to the horizon of the black hole would modify the particle wave functions near the vicinity of the horizon and thus it is possible that these divergences would be removed. This had been modeled in [10] by introducing a cut-off in the number of allowed energy levels of the quantum field near the horizon of the black hole. This approach of introducing a brick wall cut-off method has also been employed in dimensions other than $3+1[52,53]$, in order to calculate the thermodynamic quantities of interest. It was shown that the cut-off depends only on the black hole horizon in $3+1$ dimensions while it depends on the mass of the black hole as well as the mass of the quantum field in other dimensions [52]. It was also shown that the divergence in the entropy per unit area of a Klein-Gordon field propagating in the black hole background can be absorbed by renormalizing the gravitational constant $[54,55]$.

In [56], 't Hooft's approach of brick wall method was employed to calculate the entropy of BTZ black hole. The BTZ black hole is a solution to gravity in $2+1$ dimensions [57]. Gravity in $2+1$ dimensions has no propagating degrees of freedom like the $1+1$ dimensional models [58] and thus provides a good testing ground for analyzing various aspects of quantum gravity and black hole physics. In [56], entropy of a scalar theory in the background of BTZ metric was calculated using the brick wall cut-off, with appropriate choice of cut-off parameter, in the background of rotating as well as stationary BTZ black hole metrics. It was shown that the entropy in both cases is related to the perimeter of the BTZ black hole. Further, this cut-off is independent of the mass and angular momentum of the BTZ black hole.

In this paper, we probe the geometry of a BTZ black hole using a $\kappa$-deformed noncommutative scalar field as a simple probe. Using the realization of the map used in $[48,49]$, we obtain the thermodynamic properties of the BTZ black hole in $\kappa$-deformed spacetime, by analyzing the $\kappa$-deformed Klein-Gordon field in the background of the BTZ black hole. Following $[10,56]$, we calculate the entropy of the BTZ black hole in the $\kappa$-deformed spacetime by analyzing the $\kappa$-deformed Klein-Gordon field theory near the vicinity of the black hole horizon. Using the ideas developed in $[37,38,40,48,49]$, we first obtain the Klein-Gordon theory in the $2+1$ dimensional $\kappa$-deformed spacetime. Starting with the action of this model in the $\kappa$-deformed BTZ background, we calculate the free energy and entropy, semiclassically using the brick wall cut-off. We restrict our attention here only to the case of nonrotating BTZ black hole. We start with the Klein-Gordon theory in the $\kappa$-deformed BTZ background, keeping terms up to leading order in the deformation parameter. The corresponding action is shown to have higher derivative terms. Using the methods of higher derivative theories, we obtain the equations of motion for the scalar theory, which is valid up to the first order in the deformation parameter. Using the WKB method, we then calculate the energy eigenvalues of the scalar field quanta. Using this, we calculate the free energy and entropy of the system where we use the brick wall cut-off. We obtain the modification to the entropy due to the $\kappa$-deformation, valid up to first order in the deformation parameter $a$. This modification can be interpreted as renormalization of Newton's constant $G$. Noncommutativity in its most general form is introduced and presented in the Appendix. There we use a commutator between NC curved coordinates $\widehat{X}_{\mu}$ and momenta $\widehat{P}_{\mu}$ to define the NC metric. As a next step we define the general NC action. We also find the corresponding generalized equations of motion. They are valid for a generic NC scalar field $\hat{\phi}$ on a generic NC curved spacetime $\widehat{g}_{\mu \nu}$. The procedure carried out in Section 2 is actually a special case $(\mathscr{A} \neq 0$ and $\mathscr{B}=0)$ of this general procedure. We emphasize that in Section 2 we used for simplicity BTZ as a toy model and that the procedure presented in the Appendix and its special case of Section 2 are valid for a general metric $g_{\mu \nu}$.

This paper is organized as follows. In the next section, we discuss the construction of the action describing $\kappa$-deformed scalar theory with nontrivial metric background. Here, we use the $\star$-product for defining the action for $\kappa$-deformed scalar theory. We also derive the corresponding equations of motion in this section. A more general construction is discussed in the Appendix. In Section 3, we investigate $\kappa$ deformed scalar theory in the background of BTZ black hole. We calculate the entropy of the BTZ black hole using brick 
wall cut-off method. Further, we show that the modification of the entropy of the black hole due to noncommutativity of the spacetime can be absorbed by renormalizing the Newton's constant. We present our concluding remarks and discussions in Section 4. In the Appendix, we present the general construction of the action describing scalar theory in the $\kappa$-deformed spacetime with nontrivial curvature.

\section{NC Scalar Field in Curved Spacetime}

In this section we investigate $\kappa$-deformed scalar field in classical curved background. In the undeformed (commutative) spacetime, the action for scalar (Klein-Gordon (KG)) field is given by

$$
\mathcal{S}_{0}=\int d^{4} x \sqrt{-g}\left(g^{\mu \nu} \partial_{\mu} \phi \partial_{\nu} \phi-m^{2} \phi^{2}-\xi R \phi^{2}\right)
$$

and the corresponding equation of motion is

$$
\frac{1}{\sqrt{-g}} \partial_{\mu}\left(\sqrt{-g} g^{\mu \nu} \partial_{\nu} \phi\right)+m^{2} \phi+\xi R \phi=0,
$$

where $m$ is the mass of the field, $\xi$ is a parameter, and $R$ is the Ricci scalar. Here we study the generalization of (2) and (3) to NC spacetime, more specifically to $\kappa$-Minkowski spacetime.

The most natural (and easiest) way to do this is to promote the pointwise multiplication to a star multiplication in (2), that is, $f(x) g(x) \rightarrow f(x) \star g(x)$. There exists an isomorphism between NC algebra $\widehat{\mathscr{A}}$, generated by noncommutative coordinates $\widehat{x}_{\mu}$, and star algebra $\mathscr{A}^{\star}$, generated by commutative coordinates $x_{\mu}$, but with $\star$ as the algebra multiplication. Star product between any two elements $f(x)$ and $g(x)$ in $\mathscr{A}^{\star}$ is defined as

$$
f(x) \star g(x)=\widehat{f}(\widehat{x}) \widehat{g}(\widehat{x}) \triangleright 1,
$$

where $\widehat{f}(\widehat{x})$ and $\widehat{g}(\widehat{x})$ are elements of $\widehat{\mathscr{A}}$, and the action $\triangleright$ : $\mathscr{H} \mapsto \mathscr{A}$ is defined by

$$
x_{\mu} \triangleright f(x)=x_{\mu} f(x), \quad p_{\mu} \triangleright f(x)=i \frac{\partial f}{\partial x^{\mu}} .
$$

Here, $x_{\mu}$ and $p_{\mu}$ are generators of the Heisenberg algebra $\mathscr{H}$ satisfying the relations,

$$
\left[x_{\mu}, x_{\nu}\right]=\left[p_{\mu}, p_{\nu}\right]=0, \quad\left[p_{\mu}, x_{\nu}\right]=i \eta_{\mu \nu}
$$

where $\eta_{\mu \nu}=\operatorname{diag}(+,-,-,-)$ (see [51] for details on the connection between realizations, Heisenberg algebra, and star product). For simplicity we consider the case where $m=$ $\xi=0$. Thus, we postulate the following NC action $\widehat{\mathcal{S}}$ for the scalar theory in the $\kappa$-Minkowski spacetime

$$
\begin{aligned}
\widehat{\mathcal{S}} & =\int d^{4} x \sqrt{-g} g^{\mu \nu}\left(\partial_{\mu} \phi \star \partial_{\nu} \phi\right) \\
& =\int d^{4} x \sqrt{-g} g^{\mu \nu}\left(\partial_{\mu} \widehat{\phi} \partial_{\nu} \widehat{\phi} \triangleright 1\right) .
\end{aligned}
$$

Note that the gravity is treated here as classical, that is, the metric $g_{\mu \nu}$ in the above is undeformed (the procedure undertaken in this section is actually a special case $(\mathscr{A} \neq 0$ and $\mathscr{B}=0$ ), as described at the end of the Appendix). This special case consists of taking the limit of small curvatures $\left((\right.$ A.6 $\left.) \rightarrow \widehat{g}_{\mu \nu}=g_{\mu \nu}+O(a \cdot \partial g)\right)$ and of keeping only terms linear in the deformation parameter $a_{\mu}$. This means that in this section we investigate NC field $\widehat{\phi}$ on the generic classical background $g_{\mu \nu}$. Hence, in the current section we take this as our starting point when deriving (17) and (19), since our aim is to investigate the leading corrections to the entropy of BTZ, originating in noncommutativity. We emphasize that for simplicity we use here BTZ as a toy model and that the procedure presented here (as well as the procedure presented in the Appendix, which is more general one) is valid for a general metric $g_{\mu \nu}$. In the case of $\kappa$-Minkowski spacetime we can use "theory of realizations" (see [51] and references therein) and expand these star products as the power series in the deformation parameter $a_{\mu}$.

To explicitly construct the action given in (7), we first need to obtain the $\star$-product defined in (4). With this in mind, we start with the $\kappa$-Minkowski space defined by

$$
\left[\widehat{x}_{\mu}, \widehat{x}_{\nu}\right]=i\left(a_{\mu} \widehat{x}_{\nu}-a_{\nu} \widehat{x}_{\mu}\right)
$$

Operators $\widehat{x}_{\mu}$ can be realized in terms of the operators $x_{\mu}$ and $p_{\mu}\left(=i \partial_{\mu}\right)[48,49,51]$ defined in the commutative spacetime as

$$
\widehat{x}_{\mu}=x_{\alpha} \varphi_{\mu}^{\alpha}(p)
$$

Demanding consistency of this realization with (8) shows that $\varphi_{\mu}^{\alpha}(p)$ must satisfy the following conditions:

$$
\frac{\partial \varphi_{\mu}^{\alpha}}{\partial p^{\beta}} \varphi_{\nu}^{\beta}-\frac{\partial \varphi_{\nu}^{\alpha}}{\partial p^{\beta}} \varphi_{\mu}^{\beta}=a_{\mu} \varphi_{\nu}^{\alpha}-a_{\nu} \varphi_{\mu}^{\alpha}
$$

We solve (10) up to the first order in deformation parameter $a$ and get

$$
\varphi_{\mu}^{\alpha}=\delta_{\mu}^{\alpha}[1+\alpha(a \cdot p)]+\beta a^{\alpha} p_{\mu}+\gamma p^{\alpha} a_{\mu}, \quad \alpha, \beta, \gamma \in \mathbb{R},
$$

where the parameters appearing in the realization, namely, $\alpha$, $\beta$, and $\gamma$ have to satisfy the constraint

$$
\gamma-\alpha=1, \quad \beta \in \mathbb{R}
$$

This solution exhausts all possible covariant realizations up to the first order in deformation parameter $a$. The realization for an arbitrary element of $\widehat{\mathscr{A}}$, that is, $\widehat{f}$, is given by (see also [49])

$$
\begin{aligned}
\widehat{f}= & f(x)+\alpha\left(x \cdot \frac{\partial f}{\partial x}\right)(a \cdot p)+\beta(a \cdot x)\left(\frac{\partial f}{\partial x} \cdot p\right) \\
& +\gamma\left(a \cdot \frac{\partial f}{\partial x}\right)(x \cdot p)
\end{aligned}
$$


which, up to the first order in $a$, for the star product (4) yields

$$
\begin{aligned}
f(x) \star g(x)= & f(x) g(x)+i \alpha\left(x \cdot \frac{\partial f}{\partial x}\right)\left(a \cdot \frac{\partial g}{\partial x}\right) \\
& +i \beta(a \cdot x)\left(\frac{\partial f}{\partial x} \cdot \frac{\partial g}{\partial x}\right) \\
& +i \gamma\left(a \cdot \frac{\partial f}{\partial x}\right)\left(x \cdot \frac{\partial g}{\partial x}\right) .
\end{aligned}
$$

Setting $f=g=\partial \phi$ in (7) and (14), we expand the action up to the first order in the deformation parameter $a_{\mu}$ as

$$
\begin{aligned}
\widehat{\mathcal{S}}= & \mathcal{S}_{0}+\int d^{4} x \sqrt{-g} g^{\mu \nu} \\
\times & {\left[i \alpha x^{\sigma} \frac{\partial^{2} \phi}{\partial x^{\sigma} \partial x^{\mu}} a^{\beta}+i \beta(a \cdot x) \frac{\partial^{2} \phi}{\partial x_{\beta} \partial x^{\mu}}\right.} \\
& \left.+i \gamma \frac{\partial^{2} \phi}{\partial x_{\alpha} \partial x^{\mu}} a_{\alpha} x^{\beta}\right]\left(\partial_{\beta} \partial_{\nu} \phi\right) .
\end{aligned}
$$

By defining

$$
\mathscr{A}^{\alpha \beta \gamma \delta}=i \sqrt{-g} g^{\beta \delta}\left(\alpha x^{\alpha} a^{\gamma}+\beta(a \cdot x) \eta^{\alpha \gamma}+\gamma a^{\alpha} x^{\gamma}\right),
$$

we rewrite the above action in a more compact form as

$$
\widehat{\mathcal{S}}=\mathcal{S}_{0}+\int d^{4} x\left(\mathscr{A}^{\alpha \beta \gamma \delta} \frac{\partial^{2} \phi}{\partial x^{\alpha} \partial x^{\beta}} \frac{\partial^{2} \phi}{\partial x^{\gamma} \partial x^{\delta}}\right) .
$$

Starting from the noncommutative scalar theory described by the above action we derive equations of motion for the field $\phi$. Notice that the action in (17) has terms involving higher derivatives of the scalar field; that is, our Lagrangian is $\mathscr{L}=\mathscr{L}\left(\phi, \partial \phi, \partial^{2} \phi, x\right)$ and hence EulerLagrange equations will be more general as in the case of higher derivative theories. Thus the Euler-Lagrange equation relevant here is

$$
\partial_{\mu} \frac{\delta \mathscr{L}}{\delta\left(\partial_{\mu} \phi\right)}-\partial_{\mu} \partial_{\nu} \frac{\delta \mathscr{L}}{\delta\left(\partial_{\mu} \partial_{\nu} \phi\right)}=\frac{\delta \mathscr{L}}{\delta \phi} .
$$

Using this we find the Euler-Lagrange equation following from (17) explicitly as

$$
\begin{aligned}
\partial_{\sigma} & \left(\sqrt{-g} g^{\sigma \nu} \partial_{\gamma} \phi\right) \\
= & \partial_{\alpha} \partial_{\beta}\left(\mathscr{A}^{\alpha \beta \gamma \delta} \partial_{\gamma} \partial_{\delta} \phi\right)+\partial_{\gamma} \partial_{\delta}\left(\mathscr{A}^{\alpha \beta \gamma \delta} \partial_{\alpha} \partial_{\beta} \phi\right) \\
& -\frac{1}{2} \sum_{\alpha} \partial_{\alpha} \partial_{\alpha}\left(\mathscr{A}^{\alpha \alpha \gamma \delta} \partial_{\gamma} \partial_{\delta} \phi+\mathscr{A}^{\gamma \delta \alpha \alpha} \partial_{\gamma} \partial_{\delta} \phi\right) .
\end{aligned}
$$

\section{3. $\kappa$-Deformed Scalar Theory in the BTZ Background}

So far our analysis was carried out for a general curved spacetime metric $g_{\mu \nu}(x)$. Since NC effects are related to Planck scale physics, we expect that the spacetime of black holes is a natural arena for studying NC theories. Having this in mind we use the BTZ metric $[57,59]$ explicitly in $(17)$ and use the $\kappa$-deformed scalar field to probe the BTZ geometry in order to infer new features that the noncommutativity brings into the black hole physics. The BTZ black hole is described by the metric

$$
g_{\mu \nu}=\left(\begin{array}{ccc}
\frac{r^{2}}{l^{2}}-8 G M & 0 & 0 \\
0 & -\frac{1}{r^{2} / l^{2}-8 G M} & 0 \\
0 & 0 & -r^{2}
\end{array}\right) \text {, }
$$

where we have taken the angular momentum to be zero; that is, $J=0$. As said earlier, we consider NC field $\widehat{\phi}$ on the undeformed background $g_{\mu \nu}$ (we use the above metric in (16) and (17) and also choose $a_{\mu}=(a, \overrightarrow{0})$ in what follows). Even with these simplifying assumptions, the equations of motion are still nontrivial and thus we are forced to use further, physically motivated approximations. The first approximation that we take is the long wavelength limit, where we keep terms in the equations of motion that are of the lowest order in derivatives $\left(\partial \phi \gg \partial^{2} \phi, \partial^{3} \phi, \partial^{4} \phi\right)$. In this approximation the terms dependent on $\alpha$ and $\gamma$ do not contribute since they are proportional to $\partial^{(2,3,4)} \phi$, and only terms proportional to $\partial \phi$ survive. Thus, only terms depending on $\beta$ give rise to $\mathrm{NC}$ contributions. Note here that only realizations characterized with parameter $\beta$ contribute in the lowest order in the long wavelength approximation. The choice of realization corresponds to the choice of the vacuum of the theory and this should be fixed by experiment in principle. For example, $\beta=1$ corresponds to the natural realization (classical basis [51]). The equation of motion is still complicated, so we solve it using WKB approximation and obtain the spectrum. We use the ansatz $\phi(r, \theta, t)=$ $R(r) e^{-i \omega t} e^{i m \theta}$, as long as $M \gg 1$, and keep terms up to first order in the deformation parameter $a$. Taking all the above into account, we get the radial equation as

$$
\begin{aligned}
& r\left(8 G M-\frac{r^{2}}{l^{2}}\right) \frac{\partial^{2} R}{\partial r^{2}}+\left(8 G M-\frac{3 r^{2}}{l^{2}}\right) \frac{\partial R}{\partial r} \\
& +\left(\frac{m^{2}}{r}-\omega^{2} \frac{r}{r^{2} / l^{2}-8 G M}-a \beta \omega \frac{8 r}{l^{2}} \frac{3 r^{2} / 2 l^{2}-8 G M}{r^{2} / l^{2}-8 G M}\right) R=0
\end{aligned}
$$

which will be the cornerstone of the whole subsequent analyzes, presented in this paper.

3.1. Brick Wall Model and the Entropy. The calculation of entropy of the black holes using "brick wall model" was introduced in [10] for the general case and in [56] for the BTZ case. We are following the same line of arguments as $[10,56]$ 
and consequently find from (21) that the $r$-dependent radial wave number has the following form:

$$
\begin{aligned}
k^{2}(r, m, \omega)= & -\frac{m^{2}}{r^{2}\left(r^{2} / l^{2}-8 G M\right)}+\omega^{2} \frac{1}{\left(r^{2} / l^{2}-8 G M\right)^{2}} \\
& +a \beta \omega \frac{8}{l^{2}} \frac{3 r^{2} / 2 l^{2}-8 G M}{\left(r^{2} / l^{2}-8 G M\right)^{2}}
\end{aligned}
$$

where we used the ansatz $R(r)=e^{i \int k(r) \mathrm{d} r}$ and $\mathrm{WKB}$ approximation. According to the semiclassical quantization rule, the radial wave number is quantized as

$$
\pi n=\int_{r_{+}+h}^{L} k(r, m, \omega) d r,
$$

where the quantum number $n>0, m$ should be fixed such that $k(r, m, \omega)$ is real, and $h$ and $L$ are ultraviolet and infrared regulators, respectively (in the subsequent calculation for free energy and entropy we take the limit $L \rightarrow \infty$ and set $h \approx 0$ and we keep only the most divergent terms in $h$.). The total number $v$ of solutions with energy not exceeding $\omega$ is given by

$$
\nu=\sum_{-m_{0}}^{m_{0}} n=\int_{-m_{0}}^{m_{0}} d m n=\frac{1}{\pi} \int_{-m_{0}}^{m_{0}} d m \int_{r_{+}+h}^{L} k(r, m, \omega) d r .
$$

The free energy at inverse temperature $\beta_{T}$ of the black hole is

$$
\begin{aligned}
e^{-\beta_{T} F}= & \sum_{\nu} e^{-\beta_{T} E}=\prod_{\nu} \frac{1}{1-e^{-\beta_{T} E}} \\
\beta_{T} F & =\sum_{\nu} \ln \left(1-e^{-\beta_{T} E}\right) \\
& =\int d \nu \ln \left(1-e^{-\beta_{T} E}\right) \\
& =-\int_{0}^{\infty} d E \frac{\beta_{T} \nu(E)}{e^{\beta_{T} E}-1} .
\end{aligned}
$$

For this, we find the free energy $F$ as

$$
F=-\frac{1}{\pi} \int_{0}^{\infty} \frac{d \omega}{e^{\beta_{T} \omega}-1} \int_{r_{+}+h}^{L} d r \int_{-m_{0}}^{m_{0}} d m k(r, m, \omega) .
$$

After carrying out the integrations and keeping the most divergent terms in $h$, we have

$$
F=-\frac{l^{5 / 2}}{(8 G M)^{1 / 4}} \frac{\zeta(3)}{\beta_{T}^{3}} \frac{1}{\sqrt{2 h}}-2 a \beta \frac{(8 G M)^{3 / 4} \sqrt{l}}{\sqrt{2 h}} \frac{\zeta(2)}{\beta_{T}^{2}},
$$

which is the exact result in the sense of the WKB method and $\zeta$ is the Euler-Riemann zeta function.

Now we can evaluate the entropy for the NC massless scalar field using the relation $S=\beta_{T}^{2}\left(\partial F / \partial \beta_{T}\right)$. Thus we get

$$
\begin{aligned}
S & =3 \frac{l^{5 / 2}}{(8 G M)^{1 / 4}} \frac{\zeta(3)}{\beta_{T}^{2}} \frac{1}{\sqrt{2 h}}+4 a \beta \frac{(8 G M)^{3 / 4} \sqrt{l}}{\sqrt{2 h}} \frac{\zeta(2)}{\beta_{T}} \\
& =S_{0}\left(1+\frac{4}{3} a \beta \frac{8 G M}{l^{2}} \frac{\zeta(2)}{\zeta(3)} \beta_{T}\right),
\end{aligned}
$$

where $S_{0}$ is the undeformed entropy for BTZ at the Hawking temperature $\beta_{T}=2 \pi l^{2} / r_{+}$. This entropy is equivalent to the Beckenstein-Hawking entropy $S_{0}=A / 4 G=2 \pi r_{+} / 4 G$. We use this equivalence to fix the cut-off $h$ as

$$
h=\frac{9 G^{2} \zeta^{2}(3) \sqrt{8 G M}}{8 l \pi^{6}} .
$$

In other words we choose $h$ so that the entropy satisfies the area (perimeter) law, as was done in [10].

The above result can be used to obtain renormalization of the Newton's constant, which is plausible due to quantum effects at the Planck scale $[54,55]$, where noncommutative effects are expected to be important. Let the Newton's constant at the Planck scale be denoted by $G^{*}$. Assuming that the black hole area law is satisfied with the renormalized Newton's constant $G^{*}$, we get

$$
S=\frac{A}{4 G^{*}}
$$

This leads to the renormalized Newton's constant $G^{*}$ defined by

$$
\frac{1}{G^{*}}=\frac{1}{G}\left(1+\frac{8}{3} \frac{a \beta \pi}{l} \frac{\zeta(2)}{\zeta(3)} \sqrt{8 G M}\right) .
$$

Note that $G^{*}$ depends on the mass $M$ apart from $G$ and $a$ and reduces to $G$ when the noncommutative parameter $a=0$.

That the final result nevertheless reproduces the area law (30) does not look very surprising, since, indeed, the area law appears to be robust and is reproduced in various modifications (invariant or not under the local Lorentz transformations) of the field propagation (see, e.g., [60]).

\section{Final Remarks}

In this paper we have used a $\kappa$-Minkowski type noncommutative scalar field as a probe to study the BTZ black hole geometry. Using the brick wall method of [10], we have obtained the noncommutative corrections to the entropy of BTZ black holes. Our results reduce to the ones known in the literature for the pure BTZ black hole [56] when the noncommutative parameter $a$ is set to zero, as required.

Noncommutative effects are expected to be important at the Planck scale. It has been suggested that the Newton's constant may be renormalized due to quantum effects at the Planck scale $[54,55]$. Within our framework, we have calculated the noncommutative correction to the Newton's constant up to the first order in the noncommutative deformation parameter.

In this paper we have found the correction to the area law dependent on the NC parameter. This correction scales with the Beckenstein-Hawking entropy $S_{\mathrm{BH}}$ (we denote it with $S_{0}$.). This is unlike the situation which occurs in [17], where, to first order in the deformation, the noncommutative correction to the entropy has two contributions, with only one among them scaling with the Beckenstein-Hawking entropy. Moreover, this contribution that is proportional to $S_{\mathrm{BH}}$ has the negative sign, while the sign in our result depends on the $a \beta$. This 
means that the effect of noncommutativity in [17] is to reduce the number of microstates and lower the entropy and in our case noncommutativity depending on the sign of $a \beta$ will increase/decrease the number of microstates and entropy.

As it stands, our result apparently depends on the parameter $\beta$, which in turn characterizes the realization of a given noncommutative algebra (it is interesting to note that in the so-called left covariant realization (see [51] and references therein), which corresponds to Poincaré-Weyl symmetry, we have that $\beta=0$ which means that there is no NC contribution to the entropy in the leading order of the long-wavelength limit. In that case we should take even higher derivative terms into consideration, which would make the subsequent analysis much more involved.). Hence a natural question is what is the significance of the parameter $\beta$. For the $\kappa$ Minkowski space it has been clarified that to each realization there exists a particular operator ordering prescription, star product, coalgebraic structure, and a Drinfeld twist operator [40,61-64]. All these connections are realized through the one-one correspondences. In other words, the choice of the parameter $\beta$ is in one to one correspondence with the choice of the star product [40], which affects the physical consequences. It may be noted that even for Moyal spaces, the physics depends on the choice whether the Moyal product or the Voros product is used in the analysis and they lead to physically distinct predictions [65]. Thus the parameter $\beta$, through its relation to the star product, affects the physical predictions directly and empirical observations can be used to put bounds on it [64].

The system we considered can equally be viewed as two subsystems separated by the boundary area, with black hole horizon taking the role of the boundary. Then one can assign the reduced density matrix to each of the two subregions. This can be done by separately tracing the starting density matrix either with respect to the degrees of freedom of the first subregion or with respect to the degrees of freedom of the second subregion. Furthermore, if the system is to be described by a pure state, then the reduced density matrices of both subregions lead to the same entropy, which can be identified with the entanglement entropy [66]. Consequently the entanglement entropies of both subsystems are equal. In other words, the entropy of the black hole is equal to the entropy of scalar field modes propagating in and out of the black hole horizon and this is what we have calculated.

In the framework of AdS/CFT correspondence, there are arguments $[67,68]$ pointing toward the entanglement entropy as being a probe for testing the nonlocal characteristics of the theory considered. Related to AdS/CFT, two parameters, which are dual to each other, need to be specified. These are correspondingly the distance in the bulk $l$ and CFT energy scale parameter $u$. Their mutual relation (UV/IR dispersion) determines the local/nonlocal nature of the theory, with Heisenberg-like relation $l \sim 1 / u$ being the footprint of the locality, at least in the theories where the conformal symmetry is preserved.

The authors of [67] have analysed two particular theories (little string theory (LST)) and noncommutative Yang-Mills (NC YM)) and, by using their AdS/CFT description, have encountered a type of phase transition in the sense that when the characteristic size $l$ of the region considered falls well below the critical nonlocality length $l_{c}$, the area law becomes superseded by the volume law. In the case of LST, when calculating the entropy of the region of size $l$, they were able to identify two regions. In the first region (UV region), which is for $l$ much smaller than the critical size $l_{c}$, they have found the volume entropy law (cubic in $l$ or alternatively linear in $l$ if expressed in terms of the entropy density). In this region the AdS parameter $l$ does not scale with $u$, meaning that $l$ is independent of $u$ and the underlying theory is nonlocal. In the second region (IR region), that is, for large $l$, that is, $l \gg l_{c}$ and small $u$, the entropy follows the area law and the UV/IR dispersion is of the Heisenberg type $l \sim 1 / u$, indicating the locality of the underlying theory in this region [67]. Moreover, in the context of NC YM the UV/IR mixing strongly affects the critical length scale $l_{c}$ that is governing the area/volume law transition [67]. In this way entanglement entropy selects between locality and nonlocality according to whether it follows area or volume law.

In our model the characteristic size of the region considered enters the entropy through the BTZ radius $r_{+}$, which in turn enters the formula for entropy through the inverse temperature $\beta_{T}$. However, our result for the entropy has the same form for any $r_{+}$; that is, it does not depend on the characteristic size of the black hole. In this sense, while reproducing the area law, we did not come across such type of phase transition as mentioned above. Nevertheless, the analysis presented so far naturally raises the question as to why the critical value should not also exist for $r_{+}$of BTZ. Of course, we could speculate that some additional effects enter the story as $r_{+}$approaches smaller/greater values and this would certainly be an interesting question to pursue further.

At the end, we briefly comment that we are using kappa type of deformation for which UV/IR mixing is not very well understood, certainly not as good as for Moyal case, and hence the direct comparison between the two is not easy. The illumination of the role UV/IR mixing has in the context of kappa-deformation and its comparison with the Moyal case would be a possible future area of work. Furthermore, in the brick wall type calculations that we carried here, the terms dependent on the infrared cut-off are scaled away and only the most divergent term as a function of the brick wall cut-off is retained. That is what has been done here also and hence we do not see any infrared dependence. We can also argue that according to the arguments of [67], our underlying theory is local due to entropy which follows the area law.

We have used BTZ spacetime as a model for the black hole. Note that the BTZ metric appears in the discussion of near-horizon geometry of a large class of black holes [11]. Similarly, the $\kappa$-Minkowski type of noncommutativity that has been used here appears in the noncommutative description of several black hole geometries. Thus it is plausible that our results have a certain amount of universality which makes it relevant for a wider class of geometries.

\section{Appendix}

\section{NC Scalar Field in NC Curved Spacetime}

In the absence of true NC of quantum gravity theory, we have been forced to limit our analyses to the $\kappa$-deformations 
of fields and treat the gravity classically. However, in [49], authors have analyzed the most general equations of motion for particles in $\kappa$-deformed curved space. In this setting, that is, "Feynman approach," the commutator between coordinates $\widehat{X}_{\mu}$ and momenta $\widehat{P}_{\nu}$ can be interpreted as the NC metric

$$
\begin{aligned}
{\left[\widehat{X}_{\mu}, \widehat{P}_{\nu}\right] } & \equiv-i \widehat{g}_{\mu \nu} \\
& =-i g_{\alpha \beta}(\widehat{y})\left(p^{\beta} \frac{\partial \varphi_{\nu}^{\alpha}}{\partial p^{\sigma}} \varphi_{\mu}^{\sigma}+\varphi_{\nu}^{\alpha} \varphi_{\mu}^{\beta}\right),
\end{aligned}
$$

where

$$
\begin{aligned}
g_{\mu \nu}(\hat{y})= & g_{\mu \nu}(x)+\gamma\left(x \cdot \frac{\partial g_{\mu \nu}}{\partial x}\right)(a \cdot p) \\
& +\alpha\left(a \cdot \frac{\partial g_{\mu \nu}}{\partial x}\right)(x \cdot p)+\beta(x \cdot a)\left(\frac{\partial g_{\mu \nu}}{\partial x} \cdot p\right) .
\end{aligned}
$$

Equation (A.1) enables us to analyze $\kappa$-deformations of metric also. This way we can postulate the following NC covariant action (e.g., in the less complicated case of NC space called Moyal space which is defined by $\left[\widehat{x}_{\mu}, \widehat{x}_{\nu}\right]=i \Theta_{\mu \nu}$, tensor $\Theta_{\mu \nu}$ is treated as a constant tensor. In this approach (see [19-22, 69] and references therein), the symmetries are of general relativity, i.e., the diffeomorphism symmetry is formulated in the language of Hopf algebras, which provide a mathematical framework suitable for studying quantization of Lie groups and Lie algebras. A gravity theory is then constructed in such a way that it transforms covariantly under the deformed diffeomorphisms, which automatically lead to noncommutative geometry. Here it is important to introduce the notion of star product, twisted symmetries and twist operator, and their mutual relations. The construction outlined in this Appendix (as well as its special case treated in Section 2) is covariant with respect to twisted diffeomorphisms. We further emphasize that it is possible to construct the corresponding twist operator (along the line of [51], where it is shown that for every realization there is a corresponding twist operator leading to a unique associative star product) underlying the construction presented in this Appendix and which ensures the general covariance of the starting action. Here we have started from the star product and its realization. However, we could equally well start from the twist and then, using the twisted diffeomorphism, obtain the same equations of motion. The main advantage in using the star product is that the action written in terms of it is automatically covariant under the action of deformed symmetries. Of course in the limit $a_{\mu} \rightarrow 0$, the star product reduces to commutative multiplication and we recover the usual notion of covariance.) $\overrightarrow{\mathcal{S}}$ for the NC scalar theory in the $\kappa$-deformed spacetime with nontrivial metric as

$$
\begin{aligned}
\widehat{\mathcal{S}} & =\int d^{4} x \sqrt{-g}\left(g^{\mu \nu} \star \partial_{\mu} \phi \star \partial_{\nu} \phi\right) \\
& =\int d^{4} x \sqrt{-g}\left(\hat{g}^{\mu \nu} \partial_{\mu} \widehat{\phi} \partial_{\nu} \widehat{\phi} \triangleright 1\right) .
\end{aligned}
$$

In the case of $\kappa$-Minkowski spacetime we can use "theory of realizations" (see [51] and references therein) and expand these star products as power series in the deformation parameter $a_{\mu}$. Note that in the above we leave the volume $d^{4} x$ undeformed [70]. Using the $\widehat{\phi}$ and $\widehat{g}_{\mu \nu}$ (see [49] for details) valid up to first order in the deformation parameter,

$$
\begin{aligned}
\widehat{\phi}= & \phi(x)+\alpha\left(x \cdot \frac{\partial \phi}{\partial x}\right)(a \cdot p)+\beta(a \cdot x)\left(\frac{\partial \phi}{\partial x} \cdot p\right) \\
& +\gamma\left(a \cdot \frac{\partial \phi}{\partial x}\right)(x \cdot p), \\
\widehat{g}_{\mu \nu}= & g_{\mu \nu}(\widehat{y})+2 \alpha g_{\mu \nu}(a \cdot p)+\alpha g_{\nu \beta} a_{\mu} p^{\beta} \\
& +\beta\left(\eta_{\mu \nu} g_{\alpha \beta} a^{\alpha} p^{\beta}+g_{\nu \beta} a^{\beta} p_{\mu}+g_{\mu \alpha} a^{\alpha} p_{\nu}\right) \\
& +\gamma\left(g_{\mu \beta} a_{\nu} p^{\beta}+g_{\nu \beta} p^{\beta} a_{\mu}+g_{\mu \alpha} p^{\alpha} a_{\nu}\right),
\end{aligned}
$$

respectively, we now expand the action (valid up to the first order in the deformation parameter $a_{\mu}$ ). Rewriting the above equations in a more compact form as

$$
\begin{array}{r}
\widehat{g}_{\mu \nu}=g_{\mu \nu}+i a_{\alpha} G_{\mu \nu}^{\alpha \beta}(x) \partial_{\beta} \equiv g_{\mu \nu}+\delta\left(g_{\mu \nu}\right), \\
\delta\left(g_{\mu \nu}\right) \triangleright 1=0, \\
\partial_{\mu} \widehat{\phi}=\partial_{\mu} \phi+i a_{\alpha} \varphi_{\mu}^{\alpha \beta}(x) \partial_{\beta} \equiv \partial_{\mu} \phi+\delta\left(\partial_{\mu} \phi\right), \\
\delta\left(\partial_{\mu} \phi\right) \triangleright 1=0,
\end{array}
$$

we expand the action in (A.3) keeping terms up to the first order in deformation parameter. Thus we find

$$
\begin{aligned}
& \widehat{\mathcal{S}}=\int d^{4} x \sqrt{-g}\left(g_{\mu \nu}+\delta\left(g_{\mu \nu}\right)\right)\left(\partial_{\mu} \phi+\delta\left(\partial_{\mu} \phi\right)\right) \\
& \times\left(\partial_{\nu} \phi+\delta\left(\partial_{\nu} \phi\right)\right) \triangleright 1 \\
&= d^{4} x \sqrt{-g}\left(g_{\mu \nu}+\delta\left(g_{\mu \nu}\right)\right) \\
& \times\left(\partial_{\mu} \phi \partial_{\nu} \phi+\delta\left(\partial_{\mu} \phi\right) \partial_{\nu} \phi\right) \triangleright 1 \\
&= d^{4} x \sqrt{-g}\left(g_{\mu \nu}+\delta\left(g_{\mu \nu}\right)\right) \\
& \times\left(\partial_{\mu} \phi \partial_{\nu} \phi+\left[\delta\left(\partial_{\mu} \phi\right), \partial_{\nu} \phi\right]\right) \triangleright 1 \\
&= d^{4} x \sqrt{-g}\left(g^{\mu \nu} \partial_{\mu} \phi \partial_{\nu} \phi+g^{\mu \nu}\left[\delta\left(\partial_{\mu} \phi\right), \partial_{\nu} \phi\right]\right. \\
& \equiv\left.+\left[\delta\left(g_{\mu \nu}\right), \partial_{\mu} \phi \partial_{\nu} \phi\right]\right)+O\left(a^{2}\right) \\
& \mathcal{S}_{0}+a \mathcal{S}^{\prime} . \quad
\end{aligned}
$$

By using the following set of relations (note that in ((A.2)(A.9)) the $\cdot$ product is with respect to $\eta_{\mu \nu}$, so we also have $\left.g_{\mu}^{v} \equiv g_{\mu \alpha} \eta^{\alpha \beta}\right)$,

$$
\delta\left(\partial_{\mu} \phi\right)=i a_{\alpha} \varphi_{\mu}^{\alpha \beta}(x) \partial_{\beta}
$$


with

$$
\begin{aligned}
\varphi_{\mu}^{\alpha \beta}(x)= & \alpha\left(x \cdot \frac{\partial^{2} \phi}{\partial x \partial x^{\mu}}\right) \eta^{\alpha \beta}+\beta x^{\alpha} \frac{\partial^{2} \phi}{\partial x_{\beta} \partial x^{\mu}} \\
+ & \gamma \frac{\partial^{2} \phi}{\partial x_{\alpha} \partial x^{\mu}} x^{\beta}, \\
& \delta\left(g_{\mu \nu}\right)=i a_{\alpha} G_{\mu \nu}^{\alpha \beta}(x) \partial_{\beta},
\end{aligned}
$$

with

$$
\begin{aligned}
G_{\mu \nu}^{\alpha \beta}(x)= & \gamma\left(x \cdot \frac{\partial g_{\mu \nu}}{\partial x}\right) \eta^{\alpha \beta}+\alpha \frac{\partial g_{\mu \nu}}{\partial x_{\alpha}} x^{\beta}+\beta x^{\alpha} \frac{\partial g_{\mu \nu}}{\partial x_{\beta}} \\
& +2 \alpha g_{\mu \nu} \eta^{\alpha \beta}+\alpha g_{\nu}^{\beta} \delta_{\mu}^{\alpha} \\
& +\beta\left(\eta_{\mu \nu} g_{\alpha}^{\beta}+g_{\nu \alpha} \delta_{\mu}^{\beta}+g_{\mu \alpha} \delta_{\nu}^{\beta}\right) \\
& +\gamma\left(g_{\mu}^{\beta} \delta_{\nu}^{\alpha}+g_{\nu}^{\beta} \delta_{\mu}^{\alpha}+g_{\mu}^{\beta} \delta_{\nu}^{\alpha}\right),
\end{aligned}
$$

we obtain the expression for the action in (A.7) as

$$
\begin{aligned}
& \widehat{\mathcal{S}}=\mathcal{S}_{0}+\int d^{4} x \sqrt{-g} \\
& \times\left\{g ^ { \mu \nu } \left[i \alpha x^{\sigma} \frac{\partial^{2} \phi}{\partial x^{\sigma} \partial x^{\mu}} a^{\beta}+i \beta(a \cdot x) \frac{\partial^{2} \phi}{\partial x_{\beta} \partial x^{\mu}}\right.\right. \\
&\left.+i \gamma \frac{\partial^{2} \phi}{\partial x_{\alpha} \partial x^{\mu}} a_{\alpha} x^{\beta}\right]\left(\partial_{\mu} \partial_{\nu} \phi\right) \\
&\left.+i a_{\alpha} G_{\mu \nu}^{\alpha \beta}\left[\frac{\partial^{2} \phi}{\partial x^{\beta} \partial x_{\mu}} \frac{\partial \phi}{\partial x_{\nu}}+\frac{\partial^{2} \phi}{\partial x^{\beta} \partial x_{\nu}} \frac{\partial \phi}{\partial x_{\mu}}\right]\right\},
\end{aligned}
$$

which is valid up to first order in the deformation parameter. Further, by defining

$$
\begin{gathered}
\mathscr{A}^{\alpha \beta \gamma \delta}=i \sqrt{-g} g^{\beta \delta}\left(\alpha x^{\alpha} a^{\gamma}+\beta(a \cdot x) \eta^{\alpha \gamma}+\gamma a^{\alpha} x^{\gamma}\right), \\
\mathscr{B}_{\rho \sigma}^{\beta}=i \sqrt{-g} a_{\alpha}\left(G_{\rho \sigma}^{\alpha \beta}+G_{\sigma \rho}^{\alpha \beta}\right),
\end{gathered}
$$

we rewrite the above action in a more compact form as

$$
\begin{aligned}
\widehat{\mathcal{S}}= & \mathcal{S}_{0} \\
& +\int d^{4} x \\
& \times\left(\mathscr{A}^{\alpha \beta \gamma \delta} \frac{\partial^{2} \phi}{\partial x^{\alpha} \partial x^{\beta}} \frac{\partial^{2} \phi}{\partial x^{\gamma} \partial x^{\delta}}+\mathscr{B}^{\alpha \beta \gamma} \frac{\partial^{2} \phi}{\partial x^{\alpha} \partial x^{\beta}} \frac{\partial \phi}{\partial x^{\gamma}}\right) .
\end{aligned}
$$

Equation (A.11) represents the NC action for NC scalar field in NC background expanded to the first order in the deformation parameter $a$.

Starting from the noncommutative scalar field theory described by the action (A.11), we derive equations of motion for the field $\phi$. Note that the action in (A.11) has terms involving higher derivatives of the scalar field; that is, our Lagrangian is $\mathscr{L}=\mathscr{L}\left(\phi, \partial \phi, \partial^{2} \phi, x\right)$. Hence, as earlier, we derive the Euler-Lagrange equation as that in higher derivative theories. Thus the Euler-Lagrange equation relevant here is

$$
\partial_{\mu} \frac{\delta \mathscr{L}}{\delta\left(\partial_{\mu} \phi\right)}-\partial_{\mu} \partial_{\nu} \frac{\delta \mathscr{L}}{\delta\left(\partial_{\mu} \partial_{\nu} \phi\right)}=\frac{\delta \mathscr{L}}{\delta \phi} .
$$

In order to calculate equations of motion we notice that

$$
\begin{gathered}
\frac{\delta \mathscr{L}}{\delta \phi}=0, \quad \frac{\delta\left(\partial_{\alpha} \phi\right)}{\delta\left(\partial_{\mu} \phi\right)}=\delta_{\alpha}^{\mu}, \\
\frac{\delta\left(\partial_{\alpha} \partial_{\beta} \phi\right)}{\delta\left(\partial_{\mu} \partial_{\nu} \phi\right)}=\delta_{\alpha}^{\mu} \delta_{\beta}^{\nu}+\delta_{\alpha}^{\nu} \delta_{\beta}^{\mu}-\Theta_{\alpha \beta}^{\mu \nu}, \\
\frac{\delta \mathscr{L}}{\delta\left(\partial_{\sigma} \phi\right)}=2 \sqrt{-g} g_{\nu}^{\sigma} \partial^{\nu} \phi+\mathscr{B}^{\alpha \beta \sigma}\left(\partial_{\alpha} \partial_{\beta} \phi\right) \\
\frac{\delta \mathscr{L}}{\delta\left(\partial_{\sigma} \partial_{\rho} \phi\right)}=\mathscr{A}^{\alpha \beta \gamma \delta}\left[\left(\delta_{\alpha}^{\sigma} \delta_{\beta}^{\rho}+\delta_{\alpha}^{\rho} \delta_{\beta}^{\sigma}-\Theta_{\alpha \beta}^{\rho \sigma}\right)\left(\partial_{\gamma} \partial_{\delta} \phi\right)\right. \\
\left.+\left(\delta_{\gamma}^{\sigma} \delta_{\delta}^{\rho}+\delta_{\gamma}^{\rho} \delta_{\delta}^{\sigma}-\Theta_{\gamma \delta}^{\rho \sigma}\right)\left(\partial_{\alpha} \partial_{\beta} \phi\right)\right] \\
+\mathscr{B}^{\alpha \beta \gamma}\left(\delta_{\alpha}^{\sigma} \delta_{\beta}^{\rho}+\delta_{\alpha}^{\rho} \delta_{\beta}^{\sigma}-\Theta_{\alpha \beta}^{\rho \sigma}\right)\left(\partial_{\gamma} \phi\right)
\end{gathered}
$$

Using this (where we defined $\Theta_{\alpha \beta}^{\mu \nu}=1$ when $\alpha=\beta=$ $\mu=\nu$ and $\Theta_{\alpha \beta}^{\mu \nu}=0$ otherwise) we find the Euler-Lagrangian equation explicitly as

$$
\begin{aligned}
\partial_{\sigma} & \left(\sqrt{-g} g^{\sigma \nu} \partial_{\nu} \phi\right) \\
= & \partial_{\alpha} \partial_{\beta}\left(\mathscr{A}^{\alpha \beta \gamma \delta} \partial_{\gamma} \partial_{\delta} \phi\right)+\partial_{\gamma} \partial_{\delta}\left(\mathscr{A}^{\alpha \beta \gamma \delta} \partial_{\alpha} \partial_{\beta} \phi\right) \\
& +\partial_{\alpha} \partial_{\beta}\left(\mathscr{B}^{\alpha \beta \gamma} \partial_{\gamma} \phi\right)-\frac{1}{2} \partial_{\sigma}\left(\mathscr{B}^{\alpha \beta \sigma} \partial_{\alpha} \partial_{\beta} \phi\right) \\
& -\frac{1}{2} \sum_{\alpha} \partial_{\alpha} \partial_{\alpha}\left(\mathscr{A}^{\alpha \alpha \gamma \delta} \partial_{\gamma} \partial_{\delta} \phi+\mathscr{A}^{\gamma \delta \alpha \alpha} \partial_{\gamma} \partial_{\delta} \phi+\mathscr{B}^{\alpha \alpha \gamma} \partial_{\gamma} \phi\right)
\end{aligned}
$$

Though (A.14) is in principle highly nontrivial, we can separate the analysis in three different cases.

(i) NC field $\widehat{\phi}$ on undeformed background $g_{\mu \nu}$ : in this case we have $\mathscr{A} \neq 0, \mathscr{B}=0$, where $\mathscr{A}, \mathscr{B}$ are defined in (A.10a) and (A.10b).

(ii) Commutative field $\phi$ on deformed background $\widehat{g}_{\mu \nu}$ : $\mathscr{A}=0, \mathscr{B} \neq 0$,

(iii) NC field $\widehat{\phi}$ on deformed background $\widehat{g}_{\mu \nu}: \mathscr{A} \neq 0, \mathscr{B} \neq$ 0 .

We have analyzed the first situation in this paper. Other two cases are presently under investigation and will be reported elsewhere. 


\section{Conflict of Interests}

The authors declare that there is no conflict of interests regarding the publication of this paper.

\section{References}

[1] A. Connes, Noncommutative Geometry, Academic Press, New York, NY, USA, 1994.

[2] S. Doplicher, K. Fredenhagen, and J. E. Roberts, "Spacetime quantization induced by classical gravity," Physics Letters B, vol. 331, no. 1-2, pp. 39-44, 1994.

[3] S. Doplicher, K. Fredenhagen, and J. E. Roberts, "The quantum structure of spacetime at the Planck scale and quantum fields," Communications in Mathematical Physics, vol. 172, no. 1, pp. 187220, 1995.

[4] M. R. Douglas and N. A. Nekrasov, "Noncommutative field theory," Reviews of Modern Physics, vol. 73, no. 4, pp. 977-1029, 2001.

[5] R. J. Szabo, "Quantum field theory on noncommutative spaces," Physics Reports, vol. 378, no. 4, pp. 207-299, 2003.

[6] J. D. Bekenstein, "Black holes and entropy," Physical Review D, vol. 7, pp. 2333-2346, 1973.

[7] J. D. Bekenstein, "Generalized second law of thermodynamics in black-hole physics," Physical Review D, vol. 9, no. 12, pp. 32923300, 1974.

[8] S. W. Hawking, "Particle creation by black holes," Communications in Mathematical Physics, vol. 43, no. 3, pp. 199-220, 1975.

[9] W. G. Unruh, "Notes on black-hole evaporation," Physical Review D, vol. 14, no. 4, pp. 870-892, 1976.

[10] G. 't Hooft, “On the quantum structure of a black hole," Nuclear Physics B, vol. 256, no. 4, pp. 727-745, 1985.

[11] O. Aharony, S. S. Gubser, J. Maldacena, H. Ooguri, and Y. $\mathrm{Oz}$, "Large $N$ field theories, string theory and gravity," Physics Reports. A Review Section of Physics Letters, vol. 323, no. 3-4, pp. 183-386, 2000.

[12] A. Sen, "Logarithmic corrections to Schwarzschild and other non-extremal black hole entropy in different dimensions," Journal of High Energy Physics, vol. 2013, article 156, 2013.

[13] R. K. Kaul and P. Majumdar, "Logarithmic correction to the Bekenstein-Hawking entropy," Physical Review Letters, vol. 84, no. 23, pp. 5255-5257, 2000.

[14] S. Carlip, "Black hole thermodynamics and statistical mechanics," in Physics of Black Holes, vol. 769, pp. 89-123, Springer, Berlin, Germany, 2009.

[15] D. Birmingham, K. S. Gupta, and S. Sen, "Near-horizon conformal structure of black holes," Physics Letters B, vol. 505, no. 1-4, pp. 191-196, 2001.

[16] K. S. Gupta and S. Sen, "Further evidence for the conformal structure of a Schwarzschild black hole in an algebraic approach," Physics Letters B, vol. 526, no. 1-2, pp. 121-126, 2002.

[17] J. C. López-Domínguez, O. Obregón, M. Sabido, and C. Ramírez, "Towards noncommutative quantum black holes," Physical Review D, vol. 74, no. 8, Article ID 084024, 5 pages, 2006.

[18] J. C. Lopez-Dominguez, O. Obregon, and S. Zacarias, “Towards a supersymmetric generalization of the Schwarzschild black hole," Physical Review D: Particles, Fields, Gravitation, and Cosmology, vol. 80, no. 10, Article ID 104020, 2009.
[19] P. Aschieri, C. Blohmann, M. Dimitrijević, F. Meyer, P. Schupp, and J. Wess, "A gravity theory on noncommutative spaces," Classical and Quantum Gravity, vol. 22, no. 17, pp. 3511-3532, 2005.

[20] P. Aschieri, M. Dimitrijevic, F. Meyer, and J. Wess, "Noncommutative geometry and gravity," Classical and Quantum Gravity, vol. 23, no. 6, pp. 1883-1911, 2006.

[21] T. Ohl and A. Schenkel, "Symmetry reduction in twisted noncommutative gravity with applications to cosmology and black holes," Journal of High Energy Physics, vol. 2009, no. 1, article 084, 2009.

[22] T. Ohl and A. Schenkel, "Cosmological and black hole spacetimes in twisted noncommutative gravity," Journal of High Energy Physics, vol. 10, article 052, 2009.

[23] J. W. Moffat, "Noncommutative quantum gravity," Physics Letters B, vol. 491, no. 3-4, pp. 345-352, 2000.

[24] A. H. Chamseddine, "Complexified gravity in noncommutative spaces," Communications in Mathematical Physics, vol. 218, no. 2, pp. 283-292, 2001.

[25] H. Nishino and S. Rajpoot, "Teleparallel complex gravity as foundation for noncommutative gravity," Physics Letters, Section B: Nuclear, Elementary Particle and High-Energy Physics, vol. 532, no. 3-4, pp. 334-344, 2002.

[26] A. P. Balachandran, T. R. Govindarajan, K. S. Gupta, and S. Kürkçüoğlu, "Noncommutative two-dimensional gravities," Classical and Quantum Gravity, vol. 23, no. 20, pp. 5799-5810, 2006.

[27] E. Harikumar and V. O. Rivelles, "Noncommutative gravity," Classical and Quantum Gravity, vol. 23, no. 24, pp. 7551-7560, 2006.

[28] C. Bastos, O. Bertolami, N. C. Dias, and J. N. Prata, "Phase-space noncommutative quantum cosmology," Physical Review D, vol. 78, no. 2, Article ID 023516, 2008.

[29] C. Bastos, O. Bertolami, N. C. Dias, and J. N. Prata, "Black holes and phase-space noncommutativity," Physical Review D, vol. 80, no. 12, Article ID 124038, 2009.

[30] C. Bastos, O. Bertolami, N. C. Dias, and J. N. Prata, "Noncanonical phase-space noncommutativity and the Kantowski-Sachs singularity for black holes," Physical Review D, vol. 84, Article ID 024005, 2011.

[31] B. P. Dolan, K. S. Gupta, and A. Stern, "Noncommutative BTZ black hole and discrete time," Classical and Quantum Gravity, vol. 24, no. 6, pp. 1647-1655, 2007.

[32] B. P. Dolan, K. S. Gupta, and A. Stern, "Noncommutativity and quantum structure of spacetime," Journal of Physics: Conference Series, vol. 174, no. 1, Article ID 012023, 2009.

[33] P. Schupp and S. Solodukhin, "Exact black hole solutions in noncommutative gravity," http://arxiv.org/abs/0906.2724.

[34] J. Lukierski, A. Nowicki, and H. Ruegg, "New quantum Poincaré algebra and k-deformed field theory," Physics Letters B, vol. 293, no. 3-4, pp. 344-352, 1992.

[35] S. Majid, Foundations of Quantum Group Theory, Cambridge University Press, Cambridge, UK, 2000.

[36] J. Kowalski-Glikman, "Introduction to Doubly special relativity," in Planck Scale Effects in Astrophysics and Cosmology, vol. 669 of Lecture Notes in Physics, pp. 131-159, Springer, 2005.

[37] S. Meljanac and M. Stojic, "New realizations of Lie algebra kappa-deformed Euclidean space," The European Physical Journal C, vol. 47, no. 2, pp. 531-539, 2006.

[38] S. Meljanac, S. Krešić-Jurić, and M. Stojić, "Covariant realizations of kappa-deformed space," The European Physical Journal C, vol. 51, no. 1, pp. 229-240, 2007. 
[39] S. Meljanac and S. Kresic-Juric, "Noncommutative differential forms on the kappa-deformed space," Journal of Physics A: Mathematical and Theoretical, vol. 42, no. 36, Article ID 365204, 21 pages, 2009.

[40] S. Meljanac, A. Samsarov, M. Stojić, and K. S. Gupta, " $\kappa$ Minkowski spacetime and the star product realizations," The European Physical Journal C, vol. 53, no. 2, pp. 295-309, 2008.

[41] S. Meljanac and A. Samsarov, "Scalar field theory on $\kappa$ Minkowski space-time and translation and Lorentz invariance," International Journal of Modern Physics A: Particles and Fields, Gravitation, Cosmology, vol. 26, no. 7-8, pp. 1439-1468, 2011.

[42] T. R. Govindarajan, K. S. Gupta, E. Harikumar, S. Meljanac, and D. Meljanac, "Twisted statistics in $\kappa$-minkowski spacetime," Physical Review D, vol. 77, no. 10, Article ID 105010, 2008.

[43] T. R. Govindarajan, K. S. Gupta, E. Harikumar, S. Meljanac, and D. Meljanac, "Deformed oscillator algebras and QFT in $\kappa$ Minkowski spacetime," Physical Review D, vol. 80, no. 2, Article ID 025014, 2009.

[44] K. S. Gupta, S. Meljanac, and A. Samsarov, "Quantum statistics and noncommutative black holes," Physical Review D, vol. 85, no. 4, Article ID 045029, 2012.

[45] E. Harikumar, A. K. Kapoor, and R. Verma, "Uniformly accelerating observer in $\kappa$-deformed space-time," Physical Review D, vol. 86, no. 4, Article ID 045022, 6 pages, 2012.

[46] E. Harikumar and R. Verma, "Uniformly accelerated detector in the $\kappa$-deformed Dirac vacuum," Modern Physics Letters A: Particles and Fields, Gravitation, Cosmology, Nuclear Physics, vol. 28, no. 15, Article ID 1350063, 12 pages, 2013.

[47] E. Harikumar, "Maxwell's equations on the $\kappa$-Minkowski spacetime and electric-magnetic duality," Europhysics Letters, vol. 90, no. 2, Article ID 21001, 2010.

[48] E. Harikumar, T. Juric, and S. Meljanac, "Electrodynamics on $\kappa$ Minkowski space-time," Physical Review D, vol. 84, Article ID 085020, 2011.

[49] E. Harikumar, T. Jurić, and S. Meljanac, "Geodesic equation in $\kappa$-Minkowski spacetime," Physical Review D, vol. 86, Article ID 045002, 2012.

[50] T. Jurić, S. Meljanac, and R. Štrajn, " $\kappa$-Poincaré-Hopf algebra and Hopf algebroid structure of phase space from twist," Physics Letters A, vol. 377, no. 38, pp. 2472-2476, 2013.

[51] T. Jurić, S. Meljanac, and R. Štrajn, "Twists, realizations and Hopf algebroid structure of $\kappa$-deformed phase space," International Journal of Modern Physics A: Particles and Fields, Gravitation, Cosmology, vol. 29, Article ID 1450022, 32 pages, 2014.

[52] R. B. Mann, L. Tarasov, and A. Zelnikov, "Brick walls for black holes," Classical and Quantum Gravity, vol. 9, no. 6, pp. 14871494, 1992.

[53] R. B. Mann and T. G. Steele, "Thermodynamics and quantum aspects of black holes in $(1+1)$ dimensions," Classical and Quantum Gravity, vol. 9, no. 2, pp. 475-492, 1992.

[54] L. Susskind and J. Uglum, "Black hole entropy in canonical quantum gravity and superstring theory," Physical Review D, vol. 50, no. 4, pp. 2700-2711, 1994.

[55] J.-G. Demers, R. Lafrance, and R. C. Myers, "Black hole entropy without brick walls," Physical Review. D. Third Series, vol. 52, no. 4, pp. 2245-2253, 1995.

[56] S.-W. Kim, W. T. Kim, Y.-J. Park, and H. Shin, "Entropy of the BTZ black hole in 2+1 dimensions," Physics Letters B, vol. 392, no. 3-4, pp. 311-318, 1997.
[57] M. Banados, C. Teitelboim, and J. Zanelli, "Black hole in threedimensional spacetime," Physical Review Letters, vol. 69, no. 13, pp. 1849-1851, 1992.

[58] W. T. Kim and Y.-J. Park, "Stability analysis of the dilatonic black hole in two dimensions," Physics Letters B, vol. 347, pp. 217-221, 1995.

[59] D. Birmingham, "Choptuik scaling and quasinormal modes in the anti-de Sitter space/conformal-field theory correspondence," Physical Review D, vol. 64, no. 6, Article ID 064024, 5 pages, 2001.

[60] D. Nesterov and S. N. Solodukhin, "Gravitational effective action and entanglement entropy in UV modified theories with and without Lorentz symmetry," Nuclear Physics B, vol. 842, no. 2, pp. 141-171, 2011.

[61] G. Amelino-Camelia and M. Arzano, "Coproduct and star product in field theories on Lie-algebra noncommutative spacetimes," Physical Review. D. Third Series, vol. 65, no. 8, Article ID 084044, 2002.

[62] M. Dimitrijević, L. Jonke, L. Möller, E. Tsouchnika, J. Wess, and M. Wohlgenannt, "Deformed field theory on $\kappa$-spacetime," European Physical Journal C, vol. 31, no. 1, pp. 129-138, 2003.

[63] M. Dimitrijevic, L. Muller, and E. Tsouchnika, "Derivatives, forms and vector fields on the $\kappa$-deformed Euclidean space," Journal of Physics A, vol. 37, pp. 9749-9770, 2004.

[64] A. Borowiec, S. Kumar, S. Meljanac, and A. Pachol, "Constraints on the quantum gravity scale from $\kappa$-Minkowski spacetime," Europhysics Letters, vol. 92, no. 2, article 20006, 2010.

[65] A. P. Balachandran, I. Ibort, G. Marmo, and M. Martone, "Inequivalence of quantum field theories on noncommutative spacetimes: Moyal versus Wick-Voros planes," Physical Review $D$, vol. 81, no. 8, Article ID 085017, 8 pages, 2010.

[66] S. N. Solodukhin, "Entanglement entropy of black holes," Living Reviews in Relativity, vol. 14, p. 8, 2011.

[67] J. L. F. Barbón and C. A. Fuertes, "Holographic entanglement entropy probes (non)locality," Journal of High Energy Physics, 2008.

[68] J. L. F. Barbón and C. A. Fuertes, "A note on the extensivity of the holographic entanglement entropy," Journal of High Energy Physics, vol. 2008, article 053, 2008.

[69] A. Schenkel, "Noncommutative gravity and quantum field theory on noncommutative curved spacetimes," In press, http://arxiv.org/abs/1210.1115.

[70] T. Jurić, S. Meljanac, and R. Štrajn, "Universal $\kappa$-Poincaré covariant differential calculus over $\kappa$-Minkowski space," International Journal of Modern Physics A, vol. 29, no. 22, Article ID 1450121, 14 pages, 2014. 

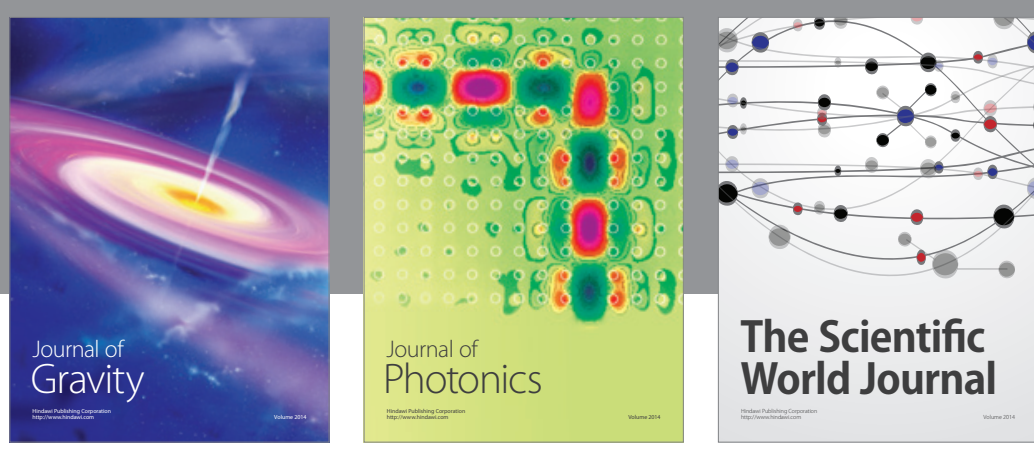

The Scientific World Journal
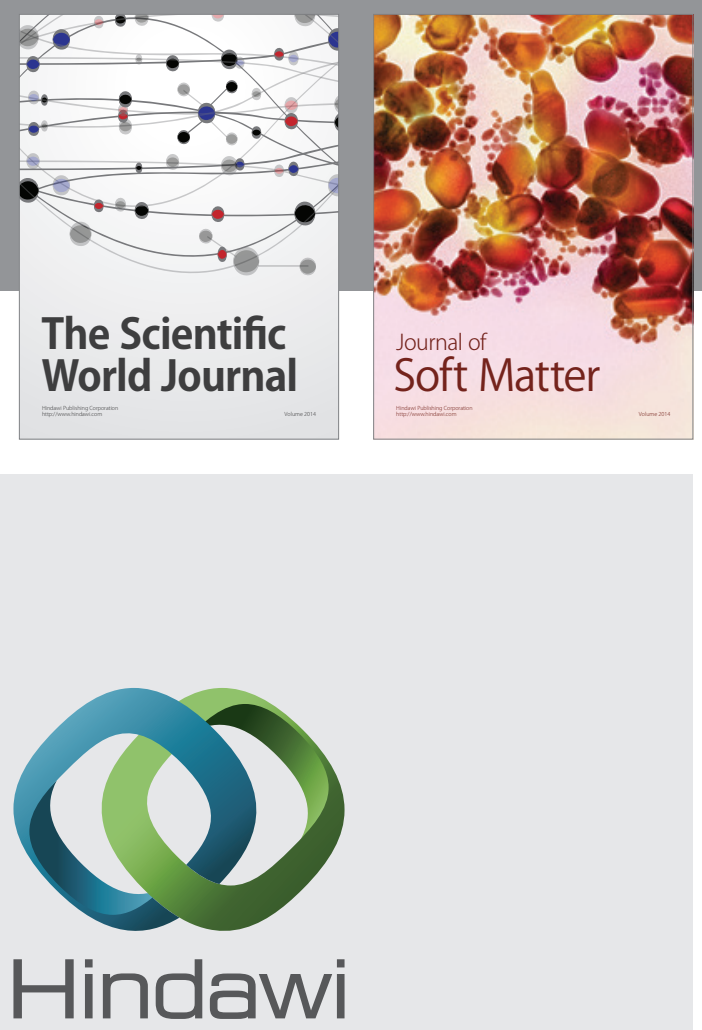

Submit your manuscripts at

http://www.hindawi.com

nternational Journal of

Statistical Mechanics
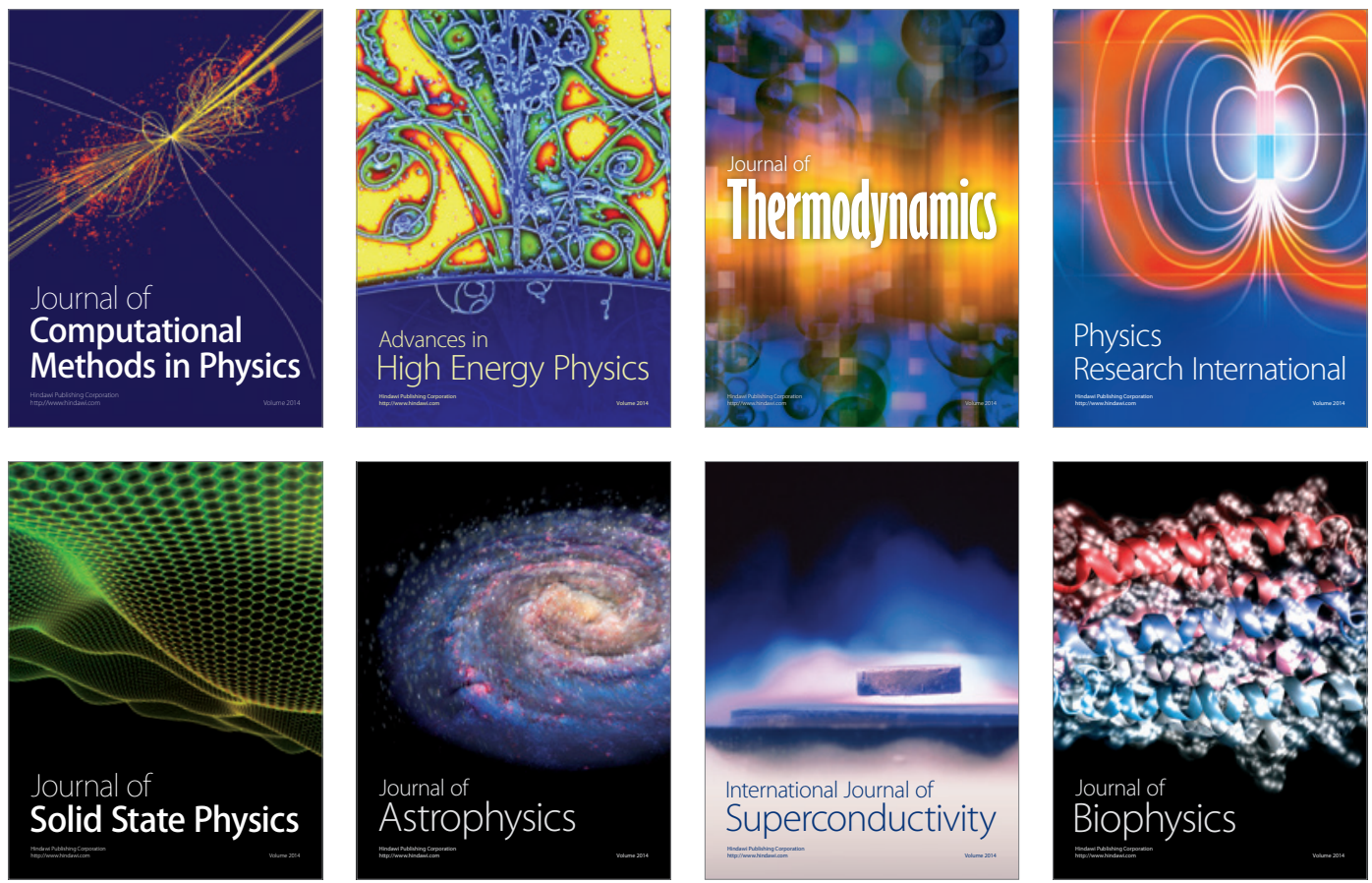
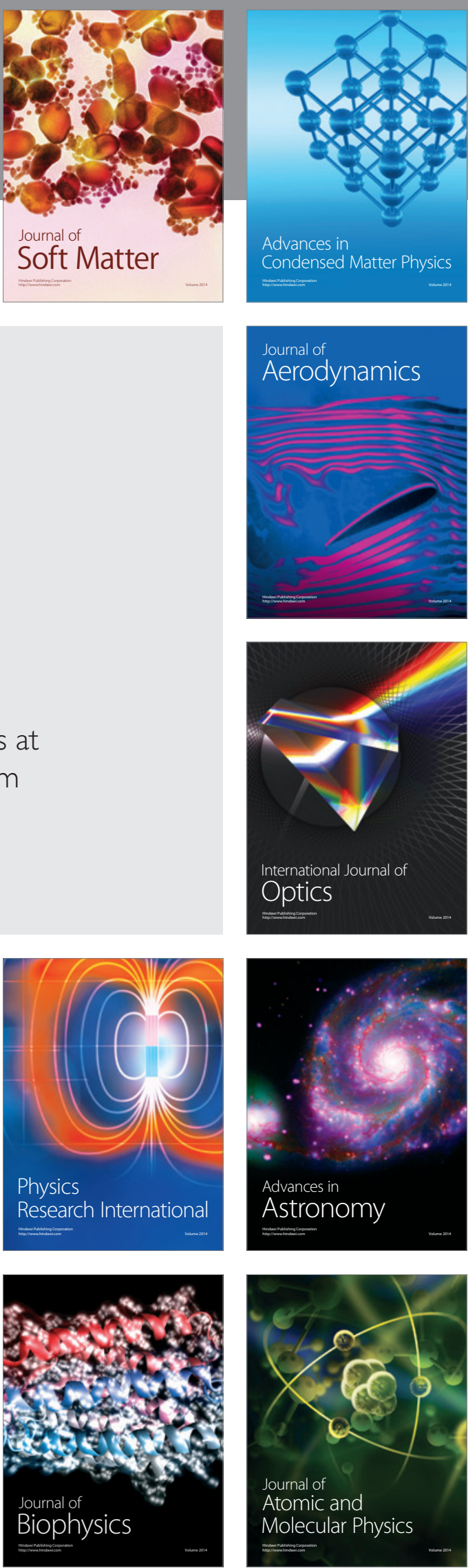